\title{
THE ELECTRON CLOUD INSTABILITY AT PEP-II
}

\author{
A. Kulikov, A. Fisher, S. Heifets, J. Seeman, M. Sullivan, U. Wienands, \\ SLAC, 2575 Sand Hill Road, Menlo Park, CA 94025, USA \\ W. Kozanecki, Saclay, France
}

\begin{abstract}
The electron cloud instability (ECI) at PEP-II enlarges the positron beam size at high currents and reduces the luminosity. The electrons for this instability come from multipacting electrons in the straight section vacuum chambers and, perhaps, photo-emission and secondary emission of electrons from the vacuum ante-chambers in the arcs. Solenoid and transverse field magnets have been placed over the vacuum chambers with about a 30 gauss field to suppress ECI. These fields have been successful in reducing the positron beam size blow up. Only about half of the drift vacuum chambers in LER have been wound with solenoids. Earlier ECI measurements are described in reference [1].
\end{abstract}

\section{LER BEAM PARAMETERS}

The Low Energy Ring (LER) of PEP-II stores positrons with a lifetime of about 200 minutes without collisions and 120 minutes during collisions [2]. Up to $2140 \mathrm{~mA}$ has been stored in LER with a typical value of about $1550 \mathrm{~mA}$ during collisions in 692 bunches. The LER lattice has a typical phase advance of 90 degrees per cell and an average beta function in both planes of about $12 \mathrm{~m}$. The horizontal and vertical emittances are about 25 and $2 \mathrm{~nm}$, respectively. The RF frequency is $476 \mathrm{MHz}$, bunches are

Table 1: LER Solenoid Parameters

$\begin{array}{lcl}\text { Parameter } & \text { LER Straights } & \text { LER Arcs } \\ \text { Chamber size (mm) } & 115 \text { dia. } & 95 \times 465 \\ \text { Wire gauge } & \# 10 & \# 10 \\ \text { Wire material } & \mathrm{Cu} & \mathrm{Cu} \\ \text { Wire package } & \text { Single cond. } & \begin{array}{l}\text { Four cond. } \\ \text { in parallel }\end{array} \\ & & 0.57 \\ \text { Solenoid field (gauss/A) } & 2.4 & \# 6 \\ \text { Return wire } & \# 10 & 200 \\ \text { Power supply voltage (V) } & 30 & 55 \\ \text { Power supply current (A) } & 12 & 31 \\ \text { Max. Field (gauss) } & 29 & 80 \\ \text { Sol. length/pow. sup. (m) } & 10-15 & 12 \\ \text { Number of power supplies } & 54 & 960 \\ \text { Available drift length (m) } & 600 & 80 \\ \text { Soln. length installed (m) } & 600 & \end{array}$

\footnotetext{
* Work supported in Department of Energy contract numbers DE-AC03-76SF00515 and DE-AC03--76-SF00098.
}

typically placed in every fourth bucket and the bunch pattern can easily be varied. If a long string of bunches with a high currents is stored in the ring, the positron beam size grows and the luminosity decreases. Solenoids have been wound over the drift sections of the beam vacuum chambers both in the straight sections and also in the bending arcs. The parameters of these solenoids are listed in Table 1.

\section{PRESSURE RISE WITH CURRENT}

The first evidence for the electron cloud effect was the correlation of the beam size blow up and multipacting in the straight sections as seen in Figure 1. Following these measurements, the straight sections were wound with solenoids. The gain in luminosity with these solenoids was very evident as described in Section 4.

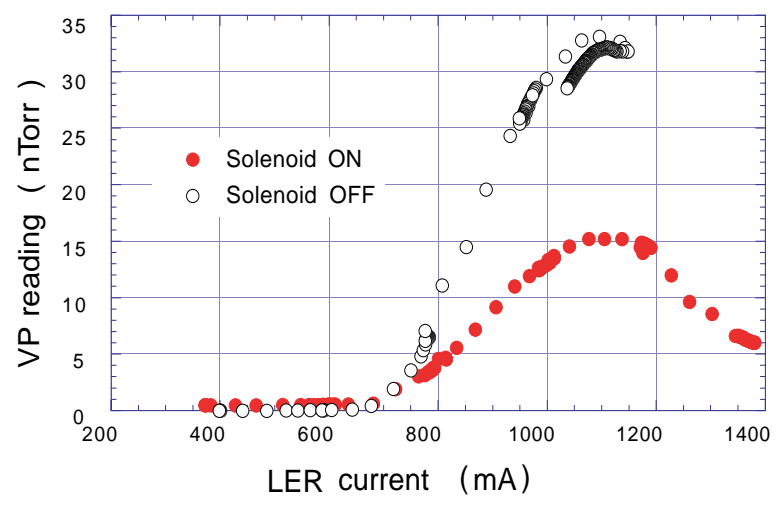

Figure 1: Electrons generated by the LER positron beam as measured by a local vacuum pump power supply and shown as pressure. The electrons come from a multipacting process driven by the positron beam and travel through the copper screens shielding the pump from the beam fields. The number of electrons generated is reduced significantly by the addition of a nearby 30 gauss solenoid field. Broad multipacting resonances can be observed in the data and predicted from a one dimensional model [3].

\section{BEAM SIZE CHANGE WITH CURRENT}

The positron beam sizes were measured as a function of stored current and the enlargement from the electron cloud 
effect is quite striking. See Figures 2 and 3. This enlargement is now believed to be a single bunch head tail instability where the very low energy electrons are captured in the positron bunch. However, a multi-bunch train is needed to produce the electron cloud.

From our observations [4] several parameters of the electron cloud have been determined. The positron bunches have about $10^{11} \mathrm{e}^{+}$each. The typical beam sizes around the ring except for the collision region are 0.75 $\mathrm{mm}$ horizontally and $0.1 \mathrm{~mm}$ vertically. Each $3.1 \mathrm{GeV}$ positron emits about 400 photons each turn for a total loss of $0.7 \mathrm{MeV}$. After a $5 \%$ bunch ring gap, the electron cloud reestablishes itself in about 120 to 400 nsec. The equilibrium time for the luminosity in a long train due to ECI is about $600 \mathrm{nsec}$. Short term resonances of the electrons can "beat' between bunches with spacings of 2 , 8 , or 16 nsec.

The estimated electron density near the positron bunch core is about 2 to $5 \times 10^{12} \mathrm{e} / \mathrm{m}^{3}$. The shift in tune spread along the train should be visible at about 0.05 to 0.07 but has not been observed so far. Each electron captured near the core of the positron beam is expected to oscillate about 2.1 times over the length of the positron bunch. The number of oscillations decreases with initial offset from the beam center. The expected peak electron velocity is about $10^{7} \mathrm{~m} / \mathrm{s}$. The expected head tail growth rate from beam-beam-up is about 15 to $25 \mu$ sec.
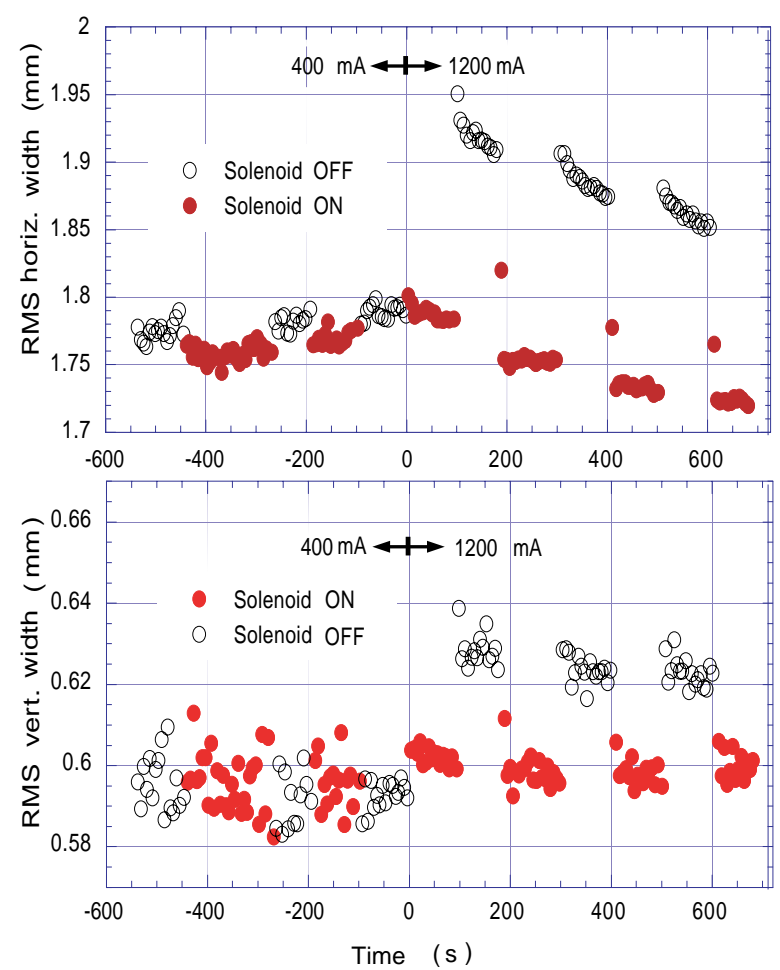

Figure 2: Horizontal and vertical beams sizes, above and below respectively, versus time at low and high LER $\mathrm{e}^{+}$ current with the solenoid on and off. At high current the solenoid has a large effect on the beam sizes driven by the electron cloud effect.
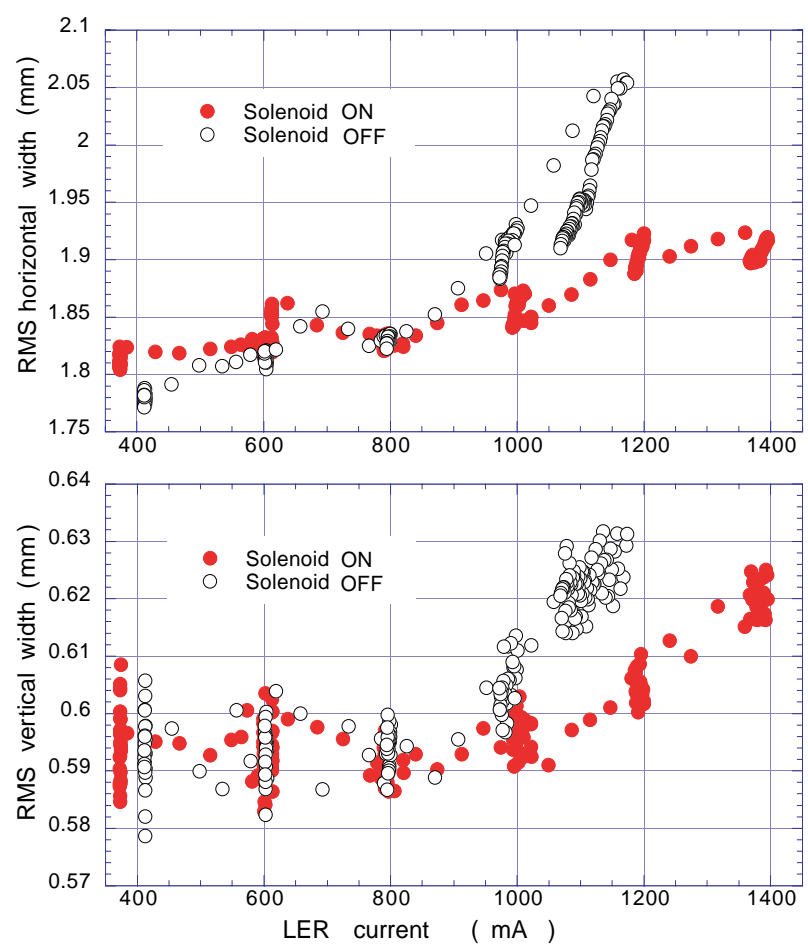

Figure 3: Horizontal and vertical beams sizes, above and below respectively, versus LER $\mathrm{e}^{+}$current with and without the solenoids. Both horizontal and vertical beam sizes grow above about $1000 \mathrm{~mA}$ without solenoids. The solenoids significantly reduce the enlargement.

\section{ELECTRON CLOUD AND LUMINOSITY}

A test pattern of long mini-trains were collided and the results shown in Figure 4. The large gap at the end of the train clears the electron cloud. The cloud slowly rebuilds itself over about $10 \%$ of the whole train. The first bunches of the mini-trains have high luminosity, which drops to $40 \%$ at the end of the train. About $200 \mathrm{~m}$ of 30 gauss solenoid in the straight sections were in operation at that time. Recently, with $400 \mathrm{~m}$ more solenoids installed, the luminosity falls only to $60 \%$ in a long train with the same bunch pattern.

During routine collisions for luminosity data taking, an additional $200 \mathrm{~m}$ of solenoid in the LER straight section was powered. The luminosity increased very quickly over a few hours to a level about $20 \%$ higher. This remarkable event is shown in Figure 5. The reason for the increase is that the threshold for the positron beam sizes to enlarge with the electron cloud was moved to higher current. The smaller beam sizes increased the luminosity. This event occurred in the summer of 2000.

In June 2001 additional data were taken when the current in $80 \mathrm{~m}$ of arc solenoid was reduced and then restored. The luminosity was recorded during this change. The results are shown in Figure 6. The luminosity decreased as the solenoid current was decreased and then 
was restored. Only minimal loss in luminosity occurred while the magnetic field was lowered from about 30 gauss ( $50 \mathrm{~A}$ ) to about 20 gauss which confirms that 30 gauss field is sufficient for effective suppression of electron multipacting. [4].

Figure 7 shows the solenoids wound in one of the LER straight sections.

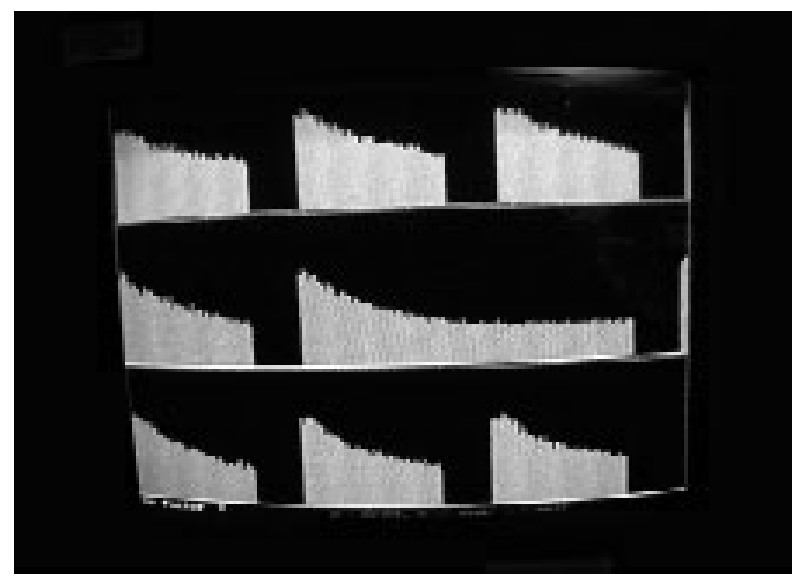

Figure 4: Test bunch pattern showing bunch-by-bunch luminosity over one turn. The build up of the electron cloud effect along the mini-trains is evident from lost luminosity. The bunch train gaps remove the electron cloud.

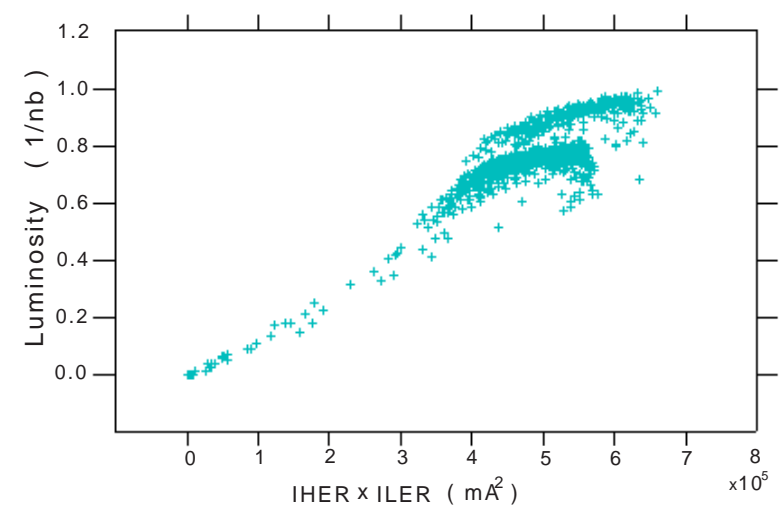

Figure 5: Luminosity versus the product of the HER and LER currents showing the luminosity gain with the addition of about $200 \mathrm{~m}$ long solenoid. The total elapsed time is about four hours and luminosity was increased by about $20 \%$.

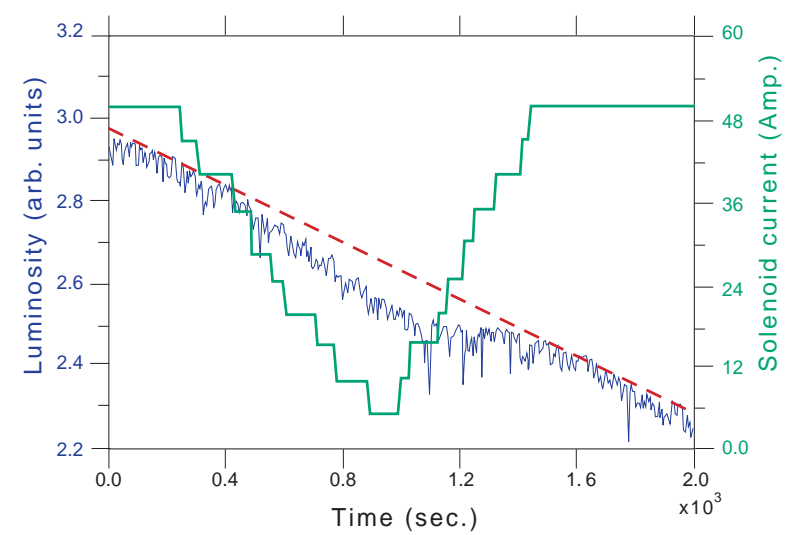

Figure 6: Luminosity and solenoid current versus time. The luminosity decreases when the magnetic field in the solenoid of $80 \mathrm{~m}$ of LER arc is decreased from the nominal 30 gauss to about 3 gauss.

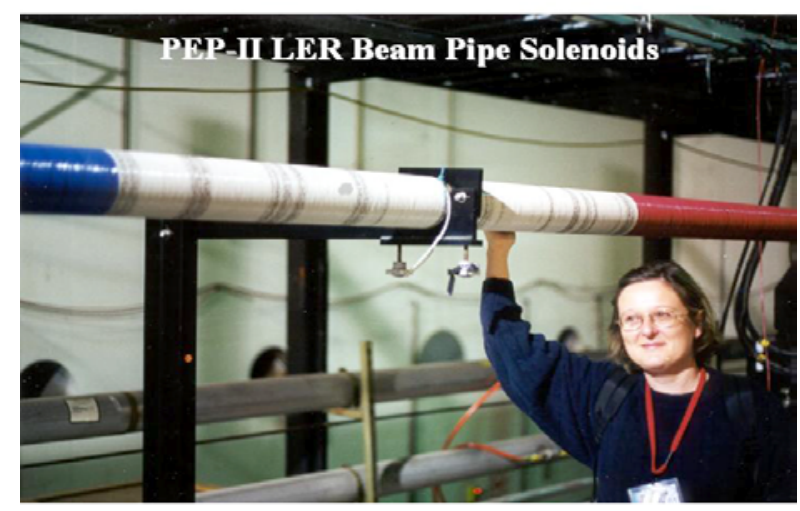

Figure 7: Straight section solenoid windings.

\section{FUTURE IMPROVEMENTS}

We have a general understanding of the electron cloud effect and instability. More measurements of the electron densities and cloud time-constants are needed. We have started a computer simulation of this effect [5]. The remaining $880 \mathrm{~m}$ of solenoid will be wound in the LER arcs as soon as repair days allow. The beam size growth with beam current will be further studied to find correlations with bunch patterns and solenoid length and strength.

\section{REFERENCES}

[1] A. Kulikov et al., "Nonlinear Pressure Rise in the PEP-II LER with Beam Current," $\mathrm{e}^{+} \mathrm{e}^{-}$Factories Workshop, KEK Proc. 99-24, p. 83.

[2] J. Seeman et al, "PEP-II Status and Plans," PAC 2001 proceedings.

[3] S. Heifets et al, SLAC-PUB-8227, 2000.

[4] J. Seeman, Proc. of the $2^{\text {nd }}$ PEP-II Performance Workshop, December 2000, p. 124.

[5] Y. Cai et al., "New Theoretical Results of the Electron Cloud Instability at PEP-II," PAC 2001 proceedings. 